\title{
From environmental stress to disease: the necessity for an integrative approach
}

\begin{abstract}
The dense network of interactions between environmental stressors and elements of the biological organization is a main challenge in the effort of elucidating the underlying mechanisms of diseases. In this context, various parameters of stress responses, like in the case of ROS homeostasis, have been described in detail. However, an integrative and holistic approach of such significant components, both as separate defense units and as part of the whole biological system is required for a more effective prevention and treatment of diseases.
\end{abstract}

Keywords: environmental stress, stress responses, ROS, epigenetics
Volume 2 Issue I - 2017

\author{
Andreas Anestis \\ Division of Technology and Sciences, The American College of \\ Thessaloniki, Greece
}

\begin{abstract}
Correspondence: Andreas Anestis, Division of Technology and Sciences, The American College of Thessaloniki, 17 Sevenidi, 55535, Pylaia, Greece, Tel (0030)-2310398343,

Email aanestis@act.edu
\end{abstract}

Received: January 13, 2017 | Published: January 25, 2017
Any effort to investigate the relation between environmental pollution and health issues brings the researchers in front of a dense and complex network of interactions and responses between environmental factors and elements of the biological organization, from genes to the whole organism. The human body, as a living system, exhibits, through a wide variety of mechanisms, a high potential to recognize environmental changes and respond to them in the appropriate way, in order to maintain its homeostasis. The ability of these mechanisms to work in a precisely organized pattern is fundamental for neutralizing the effects of disturbances that could be the cause of pathological conditions leading to diseases.

Obviously, each study, experimental or epidemiological, focuses in one or some of the branches of this network, targeting to the clarification of the relevant underlying mechanisms. However, in order for an effective prevention and treatment of diseases that have a high incidence and unclear etiology to be achieved, an integrative and holistic approach of the human body's structural and functional complexity should be adopted. Genetics, molecular biology, biochemistry, physiology but also ecology and evolution should contribute to a detailed mapping of this network of interactions, allowing the identification of the points that are crucial for maintaining the whole system within the limits of its optimum biological performance and that could be used as tools for efficiently preventing and curing diseases and estimating the relevant risk. Exposure to environmental factors and stressors is a permanent challenge for an organism. It is well known that processes like aging and neuronal degeneration have been connected with various environmental pollutants, from heavy metals and pesticides to radiation. ${ }^{1}$ Some of the basic - and in many cases common between different types of pollution-characteristics of the above network of interactions have been elucidated. For instance, in the case of atmospheric pollution, researchers have recognized that different kinds of pollutants deriving from industrial and urban activities, apart from changing the physical properties of the atmosphere, additionally function as mediators or catalysts for the formation of reactive oxygen species (ROS) within the cells.

ROS formation can be considered as being at the crossroad of a wide variety of "stress inputs", including extreme environmental temperatures, hypoxia, exposure to heavy metals and many others. ROS elevated cellular concentration is known to increase the risk for irreversible damages to macromolecules, like proteins, DNA and RNA, harming cellular physiology and leading among the others to the onset of cancer, autoimmune and neurodegenerative disorders. Cellular responses against ROS reveal an extended repertoire of mechanisms involving the activation of antioxidant enzymes, the induction of heat shock proteins (HSPs) and hypoxia induced factor (HIF) expression, the activation of signaling pathways and under severe stress the irreversible responses of autophagy, apoptosis and necrosis. ${ }^{1}$ According to a recent approach, ROS are also involved in the so called redox biology; they act as signaling molecules activating specific biological processes, when the increase of their intracellular levels is relatively small. ${ }^{2,3}$

Due to ROS central role in the development of various diseases, the diversity of ROS neutralizing mechanisms consists a typical example of a key system that should be taken in consideration in a set of different ways:

A. First, the variety of relevant defense lines reflects the evolutionary trend of all aerobic organisms to increase "gains" in the gains: losses ratio related to the necessity of using oxygen for energetically effective metabolic processes. Oxygen availability is crucial for survival, thus a decreased oxygen supply due to abnormalities in the processes of up taking, delivering to tissues and using oxygen adds a significant level of stress and can induce not only acute but also chronic disorders. ${ }^{4,5}$ At the same time, even if the above processes take normally place, the metabolic use of oxygen in the cells is accompanied by the formation of ROS as toxic metabolic byproducts. So, comparative studies examining adaptations and responses of organisms with different tolerance to decreased oxygen availability should not be ignored.

B. Second, since several responses against ROS are induced, an organization of them should be established in the cells. Of critical importance is the knowledge of the initial molecule(s) acting as a sensor of the occurring stress, the biological processes it induces, the cascade of sequential events that lead to ROS deactivation, as well as the metabolic pathways that provide the required energy. Related to the above, the "double face" of ROS as both harmful metabolic by-products and signaling molecules adds more complexity and makes obvious 
the need for determining what the critical point of transition is between their physiological and pathological role.

C. Third, the diversity of responses against ROS formation between individuals that leads to the epidemiologically confirmed differential susceptibility to diseases is a matter of genetic and epigenetic diversity involving specific genetic loci. Genetic diversity in the particular case defines the differences in the structure of genes related to ROS detoxification, while epigenetic diversity reveals the possible impact of the environment on these genes' expression. In the last years, epigenetics has been recognized as the missing link that underlies the interaction between environment and genes, especially highlighted in the case of common neurodegenerative diseases like Parkinson's and Alzheimer. ${ }^{6,7}$ The epigenetic marks, i.e. the modifications of DNA and RNA that do not affect their primary structure, act as a map showing an individual's background of specific and cumulative exposures. Epigenetic marks add a "finalizing touch" to the individuals phenotype, affecting the occurrence or not, the age of onset and the severity of a disease. Interestingly, even mild stressors and environmental factors, like nutrition, physical activity and probably vaccinations can lead to the modification of epigenetic marks. Thus, developing ways to measure genetic predisposition (as genetic makeup) and environmental exposures (as epigenetic marks) would provide strong tools for the prediction of the risk for a disease.

Despite its central role in the development of much pathology, ROS formation remains just one of the pieces that synthesize the complete picture. Approaching the biological system from a wider point of view, makes clear that what we call homeostasis or health is dependent on a complex network of such interacting "checkpoints" or equilibrium systems adjusting the biological performance close to its optimum. Working in a similar integrative way at different areas of this dense network, but also monitoring the different systems' interactions and relations as well as their overall effect on the organism's function seems to be the necessary step towards a deeper understanding of the cloudy disease mechanisms that will probably provide new targets of strategies for preventing or halting the progression of a disease.

\section{Acknowledgements}

None.

\section{Conflict of interest}

Author declares that there is no conflict of interest.

\section{References}

1. Chin-Chan M, Navarro-Yepes J, Quintanilla-Vega B. Environmental pollutants as risk factors for neurodegenerative disorders: Alzheimer and Parkinson diseases. Frontiers in cellular neuroscience. 2015.

2. Schieber M, Chandel NS. ROS function in redox signaling and oxidative stress. Curr Biol. 2014;24(10):R453-R462.

3. D'Autréaux B, Toledano MB. ROS as signaling molecules: mechanisms that generate specificity in ROS homeostasis. Nat Rev Mol Cell Biol. 2007;8(10):813-824.

4. Miyata T, Takizawa S, van Ypersele de Strihou C. Hypoxia. 1. Intracellular sensors for oxygen and oxidative stress: novel therapeutic targets. Am J Physiol Cell Physiol. 2011;300(2):C226-C231.

5. Miyata T, van Ypersele de Strihou C. Diabetic nephropathy: a disorder of oxygen metabolism? Nat Rev Nephrol. 2010;6(2):83-95.

6. Feng Y, Jankovic J, Wu YC. Epigenetic mechanisms in Parkinson's disease. J Neurol Sci. 2015;349(1-2):3-9.

7. Nicolia V, Lucarelli M, Fuso A. Environment, epigenetics and neurodegeneration: Focus on nutrition in Alzheimer's disease. Exp Gerontol. 2015;68:8-12. 